\title{
Numerical Simulation of Infrared Laser Probing Techniques
}

\author{
Robert Thalhammer, Frank Hille, Gerhard Wachutka \\ Institute for Physics of Electrotechnology, Munich University of Technology, \\ Arcisstr. 21, D-80290 Munich, Germany
}

\begin{abstract}
Exploiting the dependence of the refractive index on carrier concentration and temperature, various infrared laser probing techniques enable the experimental analysis of the carrier and heat flow distributions in the interior of semiconductor devices. In this work we present a complete numerical simulation sequence for the whole measurement process. It includes the electrothermal device simulation of the device under test, the calculation of the refractive index modulation, and the physically rigorous calculation of the propagation of the probing beam. The applicability of this method is demonstrated for the laser absorption technique.
\end{abstract}

\section{Introduction}

For the experimental analysis of the internal carrier and heat flow distributions in power devices, various infrared laser probing techniques (c. g. [1], [2]) have recently become available. All these techniques exploit the dependence of the real and imaginary part of the refractive index on carrier concentration and temperature and detect either the absorption, deflection or phase shift of an incident laser beam. For a correct interpretation of the complicated measurement signals numerical simulations are indispensable. Up to now, device simulation has been applied for the analysis of electrothermal effects which cause the refractive index modulations ([3], [4]). However, evaluation of the measurement signals is based on ray tracing and therefore neglects the effects arising from wave propagation and the lateral extension of the probing beam. For that reason, a physically rigorous method to investigate the wave propagation of the laser beam through the device is presented in this work.

\section{Simulation Steps}

Since the neglegible power dissipation of the laser beam does not change the electrothermal behavior of the device, a complete numerical simulation of the whole optical measurement process can be performed by following the simulation strategy shown in fig. 1. The special operating condition the device is subjected to during the meas-

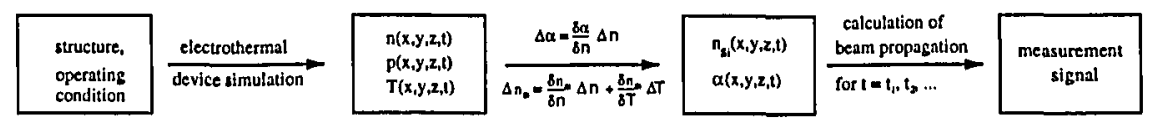

Figure 1: Simulation sequence. 
urement is investigated by electrothermal device simulation which yields the transient carrier concentration and temperature profiles. From these we obtain the spatial modulations of the real part of the refractive index $n_{S i}$ and the absorption coefficient $\alpha$. The optical module described below calculates the propagation of the laser beam at any time of interest. Calculating beam propagation at various points of time yields the time-dependent measurement signal.

\section{Electrothermal Modeling}

For accurate electrothermal modeling of devices with large power dissipation, heat generation and conduction in the semiconductor have to be treated consistently with the drift-diffusion model. Using the general purpose device simulator DESSIS ${ }^{\text {ISE }}$ [5], the device operation is simulated by solving the coupled system of Poisson's equation and the particle balances for electrons and holes together with the heat flow equation derived in [6].

\section{Refractive Index Modulation}

Since the modulations of the silicon refractive index are small, we use the linear Taylor expansions for high injection conditions $(n=p)$

$$
n_{S_{\imath}}(n=p, T)=n_{S_{t}, 0}+\frac{\partial n_{S i}}{\partial n} \Delta n+\frac{\partial n_{S i}}{\partial T} \Delta T, \quad \text { and } \quad \alpha(n=p, T)=\alpha_{0}+\frac{\partial \alpha}{\partial n} \Delta n .
$$

Our own measurements of the expansion coefficients [7] for the special operating conditions of power devices are in excellent agreement with data taken from literature ([s], [9], [10]). Note that the constant terms are not important since all the probing techniques detect the difference between on- and off-state of the device under test.

\section{Modeling of Beam Propagation}

\subsection{Basic Equation}

For the physically rigorous calculation of the laser beam propagation, we solve the 2D wave equation for the TE mode (in the coordinate system shown in fig. 2):

$$
\left(\frac{\partial^{2}}{\partial z^{2}}+\frac{\partial^{2}}{\partial x^{2}}+\frac{\omega^{2}}{c^{2}} \varepsilon_{R}\right) E_{y}(x, z)=0
$$

Finite difference discretization is applied in the propagation direction (z-axis) and the lateral $x$-direction:

$$
\begin{aligned}
0= & {\left[k_{0}^{2} \varepsilon_{R}\left(x_{j}, z_{k}\right)-\frac{1}{\Delta z_{k} \Delta \tilde{z}_{k}}-\frac{1}{\Delta z_{k} \Delta \tilde{z}_{k-1}}-\frac{1}{\Delta x_{j} \Delta \tilde{x}_{j}}-\frac{1}{\Delta x_{j} \Delta \tilde{x}_{j-1}}\right] E_{y}\left(x_{j}, z_{k}\right) } \\
& +\frac{E_{y}\left(x_{j}, z_{k+1}\right)}{\Delta z_{k} \Delta \tilde{z}_{k}}+\frac{E_{y}\left(x_{j}, z_{k-1}\right)}{\Delta z_{k} \Delta \tilde{z}_{k-1}}+\frac{E_{y}\left(x_{j+1}, z_{k}\right)}{\Delta x, \Delta \tilde{x}_{j}}+\frac{E_{y}\left(x_{\jmath-1}, z_{k}\right)}{\Delta x_{\jmath} \Delta \tilde{x}_{j-1}}
\end{aligned}
$$

\subsection{Boundary Conditions}

At the air/silicon interface $(z \nearrow 0)$, the wave propagating in the positive $z$-direction is equal to the incident wave $E_{1}$. Making use of the diffraction relations at the interface yields the boundary condition inside the sample at $z \searrow 0$ :

$$
\left(\frac{\partial}{\partial z}+i k_{z}\right) E_{y}\left(x_{j}, z_{0}\right)=2 i k_{z} E_{\imath}\left(x_{j}, z_{0}\right) \text {. }
$$

For $z=z_{\text {max }}$, a similar condition holds with $E_{i}\left(k_{x, j}, z_{\text {max }}\right)=0$, if the incident wave from the right hand side is zero. In the lateral direction, homogeneous Dirichlet boundary conditions are used to account for the vanishing electric field at the metallization layers. 
5.3. Numerical Solution

In matrix notation, equations 3 and 4 can be rewritten in the form

$$
\left(\begin{array}{ccccc}
S_{0} & D_{0} & & & \\
I & S_{1} & D_{1} & & \\
& \ddots & \ddots & \ddots & \\
& & \ddots & \ddots & \ddots \\
& & & I & S_{k_{\max }}
\end{array}\right)\left(\begin{array}{c}
\vec{u}_{0} \\
\vdots \\
\vec{u}_{k} \\
\vdots \\
\vec{u}_{k_{\max }}
\end{array}\right)=\vec{b}, \quad \vec{u}_{k}=\left(\begin{array}{c}
E_{y}\left(x_{1}, z_{k}\right) \\
\vdots \\
E_{y}\left(x_{j_{\max }}, z_{k}\right)
\end{array}\right)
$$

where $\vec{u}_{k}$ comprises the unknown fields at $z=z_{k}$. The whole matrix is a block tridiagonal matrix, where the coefficient $I$ is the unit matrix of dimension $j_{\max } \times j_{\max }, D_{k}$ are diagonal matrices and $S_{k}$ are tridiagonal matrices. Therefore, the inversion can be done very efficiently even for a large number of grid nodes. Note that equations 3 and 4 are linear, so that the solution is obtained by a single iteration.

\section{Results}

Internal carrier concentration profiles can be obtained from absorption measurements (cf. fig. 2), where the transmitted intensity of a laser beam focussed into the device is detected. The internal field distribution (cf. fig 3 ) shows standing waves in propagation direction due to multiple reflections at the polished surfaces. The lateral profile is Gaussian with the minimum spot diameter located in the center of the sample.

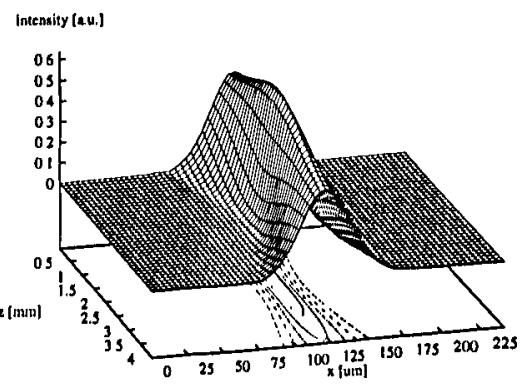

Figure 2: Experimental setup for absorption measurements.

Figure 3: Optical field distribution in the interior of the device under test (For the sake of clarity, the number of grid nodes is reduced. Standing waves are therefore not visible).

After turn-on, carrier injection causes a decrease of the transmitted intensity. Although the carrier distribution is stationary after approximately $5 \mu \mathrm{s}$, the measurement signal shows a further decrease (cf. fig. 4). This is caused by the transient temperature rise, which changes the optical interaction length by roughly $10 \%$ of a wave length and thereby the transmittance of the Fabry-Perot resonator. This is proved by the simulation with $\partial n_{S i} / \partial T=0$ (cf. Eq. 1), where a constant signal after turn-on is produced. Therefore, the carrier concentration has to be extracted from the signal right after turn-on when thermal effects are neglegible.

Assuming an exponential decrease of the transmitted intensity $I=I_{0} \exp \left(-L \frac{\partial \alpha}{\partial n} \Delta n\right)$, the carrier concentration at the vertical position of the laser beam can be calculated from the change in the transmitted intensity between on- and off-state:

$$
n_{o n} \approx n_{o n}-n_{o f f}=\frac{1}{L \frac{\partial \alpha}{\partial n}} \ln \left(\frac{I_{o f f}}{I_{o n}}\right)
$$




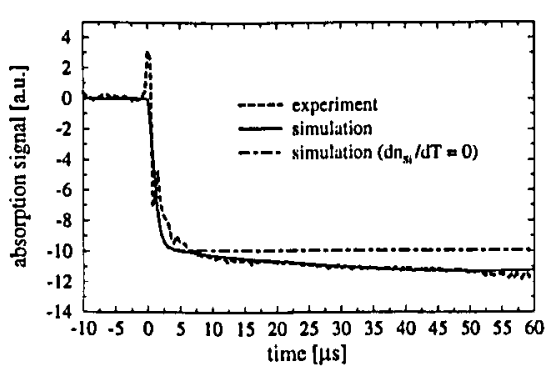

Figure 4: Transient absorption signal (change in transmitted intensity) for turn-on of a power diode.

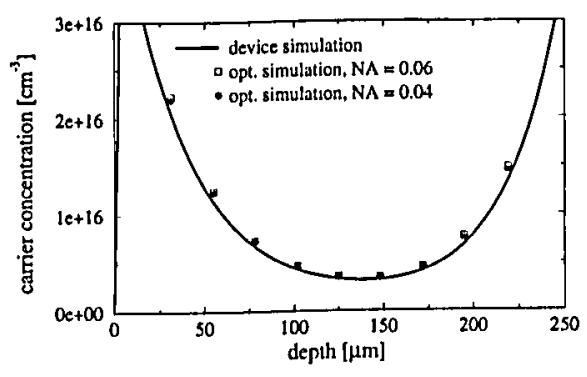

Figure 5: Carrier concentration in a pin power diode. The solid line is obtained from device simulation; dots are obtained from optic simulation and eq. 6.

Equation 6, however, neglects the averaging of the carrier density due to the lateral extension of the laser beam profile. Therefore, two beam profiles with a numerical aperture of 0.06 (76 $\mu \mathrm{m}$ maximum spot diameter) and 0.04 ( $48 \mu \mathrm{m}$ maximum spot diameter) are investigated. Figure 5 shows the comparison of the carrier distribution from electrothermal device simulation with the profile obtained from the evaluation of eq. 6. Due to the positive curvature of the carrier concentration profile, the carrier density obtained from eq. 6 is slightly higher. The error is about $10 \%$, which is in excellent agreement with the approximative error estimation presented in [7].

\section{Conclusion}

We have presented a physically rigorous method for the complete numerical simulation of infrared laser probing techniques including the calculation of the beam propagation. Accounting for wave propagation effects improves the interpretation of the measurement signals.

\section{References}

[1] G. Deboy, G. Sölkner, E. Wolfgang, W. Claeys, Microelectronic Engineering 31, 299, 1996

[2] N. Seliger, P. Ilabas, E. Gornik, Microelectronic Engineering 31, 87, 1996

[3] R. Thalhammer, G. Deboy, W. Keilitz, U. Müller, G. Wachutka, Proc. of ISDRS, Charlottesville, USA, 51, 1995

[4] R..Thalhámmer, C. Fürböck, N. Seliger, G. Deboy, E. Gornik, G. Wachutka, accepted for publication at ISPSD 98, Kyoto, Japan, 1998

[5] R. Escoffier, U. Krummbein, E. Lyumkis, B. Polsky, A. Schenk, B. Schmithüsen, C. Steiner, W. Fichtner, DESSIS 4.0 Manual, ISE AG, Zurich, CII, 1996

[6] G. Wachutka, IEEE Trans CAD, Vol. 9, No. 11, 1141, 1990

[7] F. Hille, R. Thalhammer, 'Tech. Report 1997/5, Inst. for Physics of Electrotechnology, TU Munich, 1997

[8] R. A. Soref, B. R. Bennett, IEEE J Quant Electr, Vol. 23, No. 1, 123, 1987

[9] N. Seliger, P. Ilabas, E. Gornik, Proc. of ESSDERC, Bologna, Italy, 847, 1996

[10] A. N. Magunov, J Opt. Spectroscopy, 73 (2), 205, 1992 